\title{
EQUIVALENT MODELS IN ASSOCIATION RULES ANALYSIS
}

\author{
Pannapa Changpetch ${ }^{1}$, Dennis K. J. Lin ${ }^{2}$ \\ ${ }^{1}$ Department of Mathematical Sciences, Bentley University \\ ${ }^{2}$ Department of Statistics, Pennsylvania State University
}

\begin{abstract}
A powerful methodology for exploring relationships among items, association rules analysis can be used to capture a set of rules from any given dataset. Little is known, however, that a single dataset can be represented by more than one set of rules, i.e., by equivalent models. In fact, most studies on the goodness of model can be misleading because they assume the model is unique. These are phenomenon that the literature has yet to explore. In our study, we demonstrate that equivalent models exist for any dataset and propose a method for converting any given model into its dominant model, recommended as the benchmark model. Further, we explain how the phenomenon of equivalent models affects decision tree analysis and statistical model selection. It is shown that the decision rules from decision tree analysis can always be simplified by reducing the decision rules to the dominant model. The simulated and real datasets are used for illustration.
\end{abstract}

Keywords: Association rules analysis, Decision tree analysis, Dominant model, Equivalent models.

\section{Introduction}

Association rules analysis, introduced in the early 1990s (Agrawal and Srikant 1994), is a popular data-mining technique. It is a methodology for exploring relationships among items (variables) in terms of rules. The basic idea is straightforward: to determine the rules that are effective in explaining a given dataset. These rules must meet some criteria, for example, a minimum support and a minimum confidence. Numerous studies make use of association rules analysis in a wide variety of areas, including biology (Becquet et al. 2002; Kuo et al. 2007), business and marketing (Wang et al. 2005; Shih et al. 2010), geography (Appice et al. 2003; Lee et al. 2007), agriculture (Matsumoto 1998; Mclver and Friedl 2002), education (Garcia et al. 2007; Merceron and Yacef 2008), photography (Tesic 2003; Liu et al. 2008), and economics (Dopfer and Potts 2004; Mosson et al. 2008). Many advances have been made, including methods for rules selection that prefer some rules over others based on given criteria and methods for selecting sets of rules to represent datasets (Bayardo 1997; Liu et al. 1998; Dong et al. 1999; Bastide et al. 2000; Yin and Han 2003; Webb and Zhang 2005; Li 2006; Thabtah 2007; Grudzinski et al. 2010).

However, there is a fundamental phenomenon that has been ignored which may lead to an inappropriate comparison between rules selection methodologies. This phenomenon is that of 
"equivalent models," whereby a dataset can be perfectly represented by two or more association rules models.

In the literature (Etchells and Lisboa 2006; Guerreiro and Trigueiros 2010; Marghny and El-Semman 2005), a dataset is generated via a known or given model; i.e., each methodology under consideration is tested using the generated dataset. The set of rules in the given model are the only rules considered to be correct. Any methodology that finds the same rules as those of the given model is considered to be competent. On the other hand, any methodology that finds rules that differ from those of the given model is considered to be incompetent.

Such a comparison may not be valid, however, as illustrated by the following example. Suppose that the dataset under consideration is generated via the given model, which consists of two rules:

Rule 1: If $\mathrm{X} 1=1$, then $\mathrm{Y}=1$.

Rule 2: If $\mathrm{X} 2=1, \mathrm{X} 3=1$, and $\mathrm{X} 4=1$, then $\mathrm{Y}=1$.

Consider the following two models ( $\mathrm{U}$ and $\mathrm{V})$ :

Model U:

Rule U1: If $\mathrm{X} 1=1$, then $\mathrm{Y}=1$.

Rule U2: If $\mathrm{X} 2=1, \mathrm{X} 3=1$, and $\mathrm{X} 4=1$, then $\mathrm{Y}=1$.

Model V:

Rule V1: If $\mathrm{X} 1=1$ and $\mathrm{X} 3=0$, then $\mathrm{Y}=1$.

Rule V2: If $\mathrm{X} 1=1$ and $\mathrm{X} 4=1$, then $\mathrm{Y}=1$.

Rule V3: If $\mathrm{X} 1=1, \mathrm{X} 2=1$, and $\mathrm{X} 3=1$, then $\mathrm{Y}=1$.

Rule V4: If $\mathrm{X} 1=0, \mathrm{X} 2=1, \mathrm{X} 3=1$, and $\mathrm{X} 4=1$, then $\mathrm{Y}=1$.

Rule V5: If $\mathrm{X} 1=1, \mathrm{X} 2=0, \mathrm{X} 3=1$, and $\mathrm{X} 4=0$, then $\mathrm{Y}=1$.

The responses generated by Model $\mathrm{U}$ and Model $\mathrm{V}$ are identical. In other words, the two models are equivalent. If the equivalence of models is not fully understood, comparisons among methods and among criteria can be misleading. This phenomenon has yet to be explored in the literature.

The next issue pertains to explaining the dataset or explaining the relationships among variables if there are multiple models to choose from. Our major finding on this point is that although a large number of given models can represent the same dataset; there is one unique "dominant model." We propose the dominant model as the benchmark for explaining the relationships between variables among all the equivalent models. By using the dominant model, we generate an explanation of the relationships among the variables that is consistent and concise. In the other word, the dominant model provides the better explanation for the data while keeping the same prediction ability compared to the other equivalent models. The dominant model can also be used to verify equivalency among the given models, thereby eliminating inappropriate comparisons among the rules selection methodologies. In the other word, all the models have their dominant models. If any two or more models have the same dominant model, those models are equivalent.

The effect of equivalent models is not limited to association rules analysis. In particular, the effect of equivalent models also applies to decision tree analysis. A decision tree analysis is 
characterized by a hierarchical pattern, such that any set of decision rules can generally be reduced to a set of more compact rules. Furthermore, the effect of equivalent models also applies to statistical techniques that use variables generated from decision tree analysis in order to develop a logistic regression model. We show that the decision rules from decision tree analysis and the consequent variables can always be simplified by reducing the decision rules to the dominant model.

The goal here is to explore this equivalency phenomenon. We discuss all the essential ideas and analysis including the definitions, the dominant model, the verification, and the theoretical properties of equivalent models. We show the direct effect of equivalent models on association rules analysis, decision tree analysis and on statistical model selection methods.

This paper is organized as follows. Section 2 discusses the basic concept of equivalent models through an example. Section 3 proposes the dominant model for equivalent models and the derivation to obtain the dominant model. Section 4 describes the theoretical properties of the equivalent models. Section 5 demonstrates how this phenomenon is related to the well-known MONK's dataset. Section 6 demonstrates the effect of equivalent models with real-life application. Section 7 explains the parallel effect of equivalent models on decision tree analysis and demonstrates how this phenomenon affects real-life application. Section 8 offers a discussion and concluding remarks.

\section{An illustrative example}

First, we illustrate the equivalency phenomenon of association rules analysis through an example showing that multiple models can be used to represent the same dataset. Note that this phenomenon is very common but has never been acknowledged in literature.

Consider an example with five binary variables (X1-X5) and a binary response (Y) with classes 0 and 1. Model A, which consists of two rules, is used to generate response $\mathrm{Y}$ :

Rule A1: If $\mathrm{X} 1=1$, then $\mathrm{Y}=1$.

Rule A2: If $\mathrm{X} 2=1$ and $\mathrm{X} 3=1$, then $\mathrm{Y}=1$.

The generated dataset can be used to compare the methodologies in terms of the respective performance of each. For this dataset, any technique that finds both Rule A1 and Rule A2 is considered competent. Now consider Model B, which consists of 4 rules:

Rule B1: If $\mathrm{X} 1=1$ and $\mathrm{X} 2=1$, then $\mathrm{Y}=1$.

Rule B2: If $\mathrm{X} 1=1$ and $\mathrm{X} 3=1$, then $\mathrm{Y}=1$.

Rule B3: If $\mathrm{X} 1=1, \mathrm{X} 2=0$, and $\mathrm{X} 3=0$, then $\mathrm{Y}=1$.

Rule B4: If $\mathrm{X} 1=0, \mathrm{X} 2=1$, and $\mathrm{X} 3=1$, then $\mathrm{Y}=1$.

Based on the current prevailing methodology, Model B would be considered incompetent because it did not find any rule in the given model (Model A). However, Model B generates responses that are identical to those produced by Model A. In other words, these two models are "equivalent." A lack of knowledge about model equivalence is likely to result in misleading comparisons between methods and rules selection criteria. Note that all the models shown in 
Table 1 will generate responses that are identical to those generated by Model A and Model B, such that all of these models are equivalent.

Table 1: Illustrated Equivalent Models

\begin{tabular}{|l|l|}
\hline Model & Rules \\
\hline C & $\begin{array}{l}\text { Rule C1: If } \mathrm{X}_{1}=0 \text { and } \mathrm{X}_{2}=0, \text { then } \mathrm{Y}=0 . \\
\text { Rule C2: If } \mathrm{X}_{1}=0 \text { and } \mathrm{X}_{3}=0, \text { then } \mathrm{Y}=0 .\end{array}$ \\
\hline $\mathrm{D}$ & $\begin{array}{l}\text { Rule D1: If } \mathrm{X}_{1}=1, \text { then } \mathrm{Y}=1 . \\
\text { Rule D2: If } \mathrm{X}_{1}=0, \mathrm{X}_{2}=1, \text { and } \mathrm{X}_{3}=1, \text { then } \mathrm{Y}=1 .\end{array}$ \\
\hline $\mathrm{E}$ & $\begin{array}{l}\text { Rule E1: If } \mathrm{X}_{1}=0 \text { and } \mathrm{X}_{2}=0, \text { then } \mathrm{Y}=0 . \\
\text { Rule E2: If } \mathrm{X}_{1}=0, \mathrm{X}_{2}=1, \text { and } \mathrm{X}_{3}=0, \text { then } \mathrm{Y}=0 .\end{array}$ \\
\hline $\mathrm{F}$ & $\begin{array}{l}\text { Rule F1: If } \mathrm{X}_{1}=1 \text { and } \mathrm{X}_{4}=0, \text { then } \mathrm{Y}=1 . \\
\text { Rule F2: If } \mathrm{X}_{1}=1 \text { and } \mathrm{X}_{4}=1, \text { then } \mathrm{Y}=1 . \\
\text { Rule F3: If } \mathrm{X}_{2}=1 \text { and } \mathrm{X}_{3}=1, \text { then } \mathrm{Y}=1 .\end{array}$ \\
\hline
\end{tabular}

It is evident that given differences between the terms and the number of rules between these equivalents models (Models A-F), that each model explains the relationships among the variables differently. This becomes another issue associated with equivalent models. Although it is possible, as shown, for a large number of models to represent the same dataset, there is only one unique dominant model to which all the equivalent models can refer. In fact, the dominant model serves as a point of reference whereby the explanation of the relationships among the variables will be consistent and concise. The dominant model can also be used to verify equivalency among models in order to eliminate unfair comparisons among the rules selection methodologies and criteria. The next section presents a thorough study of equivalent models including important definitions related to model equivalency. The concept of a dominant model as a representation of equivalent models is proposed, and the procedure for converting equivalent models into the dominant model is described.

\section{Equivalent Models and the Dominant Model}

This section provides a method for converting any given model into its dominant model. First, we provide definitions of all the significant terms used. Then, we propose a method for converting any given models into the dominant model. Note that the dominant model can also be used to verify equivalency among models, as models that have the same dominant model are all equivalent.

\subsection{Definitions}

Definition 1: Models are called equivalent if they generate identical responses.

Note that Models A-F in Section 2 are all equivalent. For simplicity, our study separates the equivalent models into two classes of response: class 0 and class 1 . The equivalent model with all the rules giving conditions for $\mathrm{Y}=0$ is called the equivalent model for class 0 . Likewise, the 
equivalent model with all the rules giving conditions for $\mathrm{Y}=1$ is called the equivalent model for class 1 . Note that equivalent models from one class can be viewed as complementing the equivalent models of the other class, as will be discussed later.

Definition 2: Of all the equivalent models, the dominant model is the model with the fewest condition terms.

A condition term is represented by one variable and its level, e.g., $\mathrm{Xi}=1$. We count all the repeated condition terms. As there are two classes for a binary response, there are two dominant models - one dominant model for each class. Note that the dominant model for each class is unique. The proof is provided in Section 4.

Consider the example in Section 2: Model A is the dominant model for class 1, and Model C is the dominant model for class 0 . For Model A, the total number of condition terms is three: one term from Rule A1 (X1 = 1), and two terms from Rule A2 (X2 = 1 and X3 = 1). For Model C, the total number of condition terms is four: two terms from Rule $\mathrm{C} 1(\mathrm{X} 1=0$ and $\mathrm{X} 2=0)$, and two terms from Rule $\mathrm{C} 2(\mathrm{X} 1=0$ and $\mathrm{X} 3=0)$. Note that the dominant model is determined not by the total number of distinct terms but by the total number of relevant terms that cannot be eliminated.

Definition 3: Dominant rules are all the rules in the dominant model.

As shown in Section 2, Rules A1, and A2 are dominant rules for class 1, while Rules C1 and $\mathrm{C} 2$ are dominant rules for class 0.

Definition 4: A disjoint model is a model wherein there are no rules with overlapping condition spaces.

Models D and E are disjoint models. However, Model A is not a disjoint model, as it includes instances that satisfy both Rule A1 and Rule A2. Figure 1a shows that the respective condition spaces of Rule A1 and Rule A2 overlap with each other. On the other hand, Figure 1b shows that there is no overlapping space between Rule D1 and Rule D2.

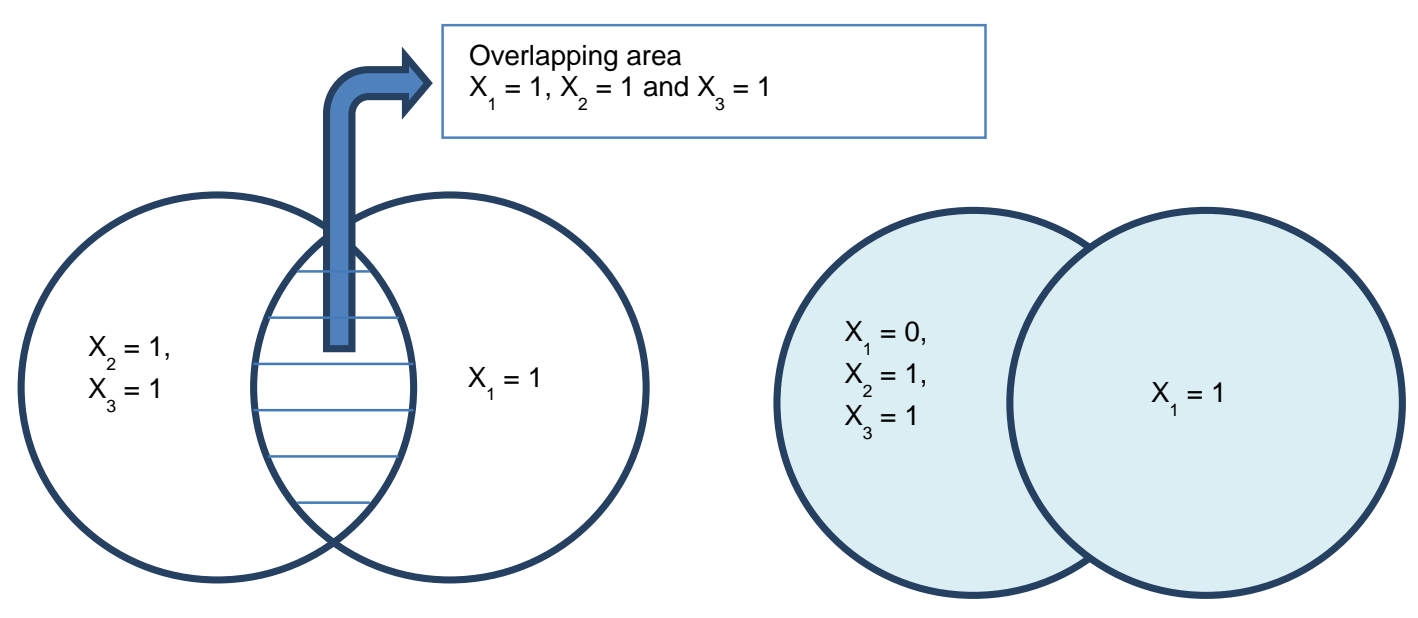

Figure 1a. Dominant Model

Figure 1b. Disjoint Model 
Definition 5: A child rule is a rule whose condition is the subset of another rule, i.e., (the parent rule).

Consider Model A and Model B in Section 2: Rule A1 is the parent rule of Rules B1, B2, and B3, whereas Rule A2 is the parent rule of Rule B4. In other words, Rules B1, B2, and B3 are the child rules of Rule A1, whereas Rule B4 is the child rule of Rule A2.

Definition 6: A complement model represents a class that differs from that of the given model.

If the given model is the model for $\mathrm{Y}=1$, then the complement model will be the model for $\mathrm{Y}=0$. For example, Model C in Section 2 is the complement model of Model A and vice versa. Definition 7: An irrelevant rule is any rule that if eliminated would not change the response.

Definition 8: An irrelevant term is any term in the condition of the rule that if eliminated would not change the response.

Consider Model F in Section 2: If we eliminate term X4 = 0 in Rule F1 and term X4 = 1 in Rule F2, the response would still be the same as it would had these terms not been eliminated. (An explanation is given in the following section.) Thus, both of these terms are irrelevant terms.

Next, we demonstrate how to convert any equivalent models into the dominant model. 


\subsection{Converting to the Dominant Model}

The dominant model is unique and it is the model with the fewest condition terms that can be used to explain a dataset. Here, we propose the dominant model as a benchmark model for all the equivalent models. A step-by-step procedure for obtaining the dominant model from any equivalent models is given at the end of this subsection.

\section{Terms from partitioned rule(s)}

Suppose there are two rules: "if $M_{1}$ and $M_{2}$, then $\mathrm{Y}=1$ " and "if $M_{1}$ and $M_{2}{ }^{c}$, then $\mathrm{Y}=1$," where $M_{2}{ }^{c}$ is the complement term of $M_{2}$. The combination of these two rules is equivalent to the rule "if $M_{1}$, then $\mathrm{Y}=1$." As we can eliminate $M_{2}$ and $M_{2}{ }^{c}$ without changing the response, both are considered irrelevant terms.

As the example in Table 2 indicates, adding $X_{4}=0$ to Rule A1 generates Rule F1. Similarly, adding $\mathrm{X}_{4}=1$ to Rule A1 generates Rule F2. Therefore, Model F is equivalent to Model A.
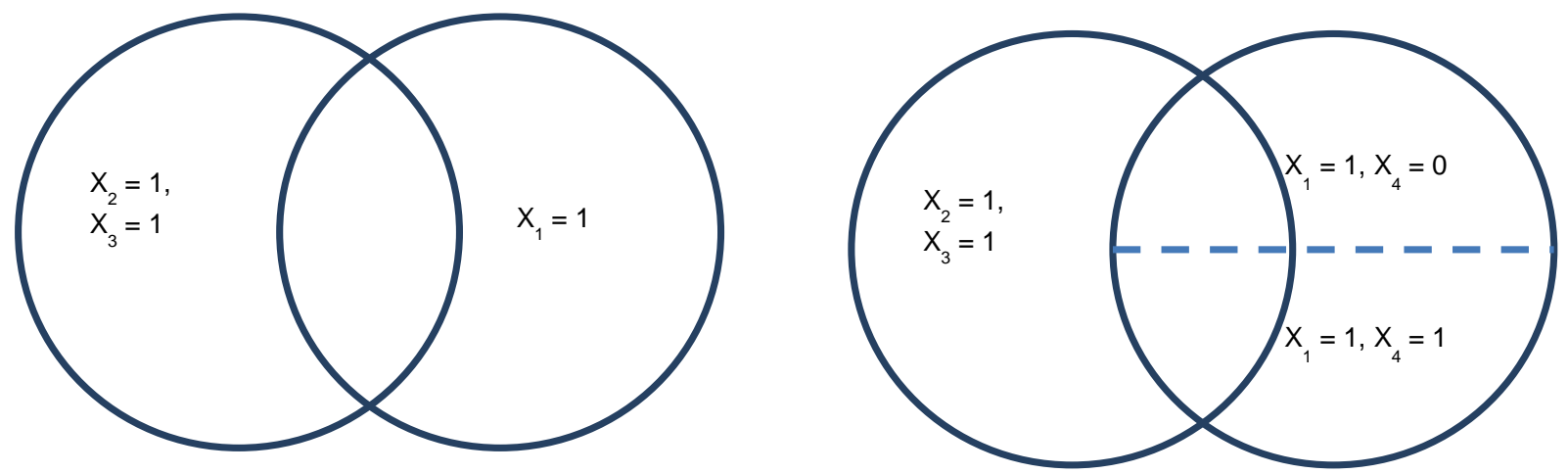

Figure 2a: Model A

Figure 2b: Model F

As shown in Figure 2, one rule can be partitioned into multiple rules. It is, therefore, possible to generate numerous equivalent models as one rule can be partitioned in multiple ways.

\section{Complement terms}

Suppose the first rule is "if $M_{l}$, then $\mathrm{Y}=1$ " and the second rule is "if $M_{2}$ and $M_{1}{ }^{c}$, then $\mathrm{Y}=$ 1 ," where $M_{1}{ }^{c}$ is the complement term of $M_{1}$. Note that the term $M_{1}{ }^{c}$ is irrelevant because it can be eliminated without changing the response. Consider the model condition of Rule D in Table 2: the complement A1 is $\mathrm{X}_{1}=0$. This term in Rule D2 can be eliminated without changing the response of the model. Some examples of irrelevant terms related to this category are shown in Figure 3. 


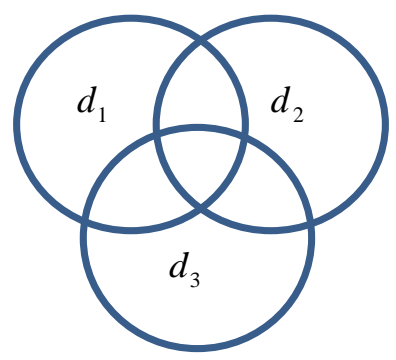

Figure 3a: Model with overlapping spaces
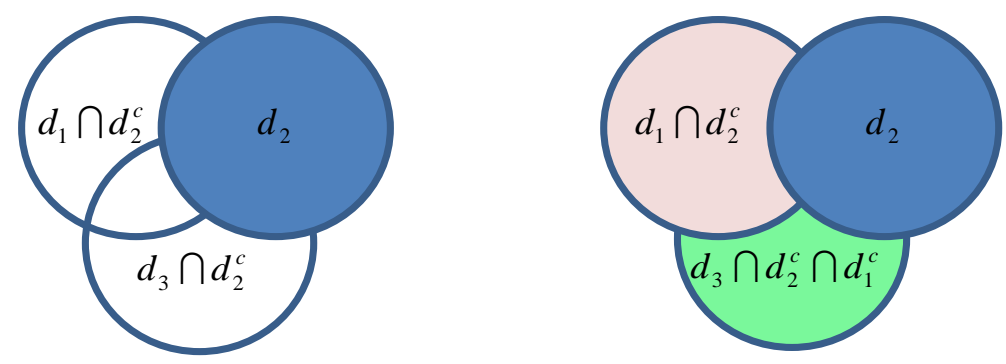

Figure 3b: Model wit Figure 3c: Model without less overlapping spaces overlapping spaces

In Figure 3a, the model without any irrelevant terms is represented with three rules. The term $d_{2}{ }^{c}$ in Figure $3 \mathrm{~b}$ and the terms $d_{1}{ }^{c}$ and $d_{2}{ }^{c}$ in Figure $3 \mathrm{c}$ are all irrelevant terms, as any of them can be eliminated without changing the response. Note that Figure $3 \mathrm{c}$ represents the disjoint model without any overlapping condition spaces.

\section{Child Rules}

Suppose we have two rules: "if $M_{l}$, then $\mathrm{Y}=1$," and "if $M_{l}$ and $M_{2}$, then $\mathrm{Y}=1$." The combination of these two rules is equivalent to the rule "if $M_{l}$, then $\mathrm{Y}=1$." Thus, the second rule is the child rule of the first rule and can be eliminated without changing the response. The rule "if $M_{1}$ and $M_{2}$, then $\mathrm{Y}=1$ " is an irrelevant rule.

\section{Procedure for the Dominant Model}

The dominant model can be obtained by deleting all the irrelevant terms and rules. We propose the iterative method for eliminating irrelevant rules and irrelevant terms as follows (also refer to Figure 4).

Step 1: Eliminate irrelevant rules

Suppose that there are k rules at the initial stage. Search for a rule that can be deleted while retaining the identical response under the remaining $\mathrm{k}-1$ rules. If such a rule is found, it is eliminated. Repeat step 1 until no more irrelevant rules are found.

\section{Step 2: Eliminate an irrelevant term}

From the model obtained in step 1, search for the condition term that can be deleted while the identical response is retained. If such a term is found, delete it from the corresponding rule, and return to step 1. (Note that only one term should be deleted from only one rule in each iteration of step 2.) If no irrelevant term can be found, the dominant model has been obtained.

\section{Remark.}


The process stops when there are no longer any irrelevant terms in the model. The form after all the irrelevant rules and all the irrelevant terms have been deleted is the dominant model.

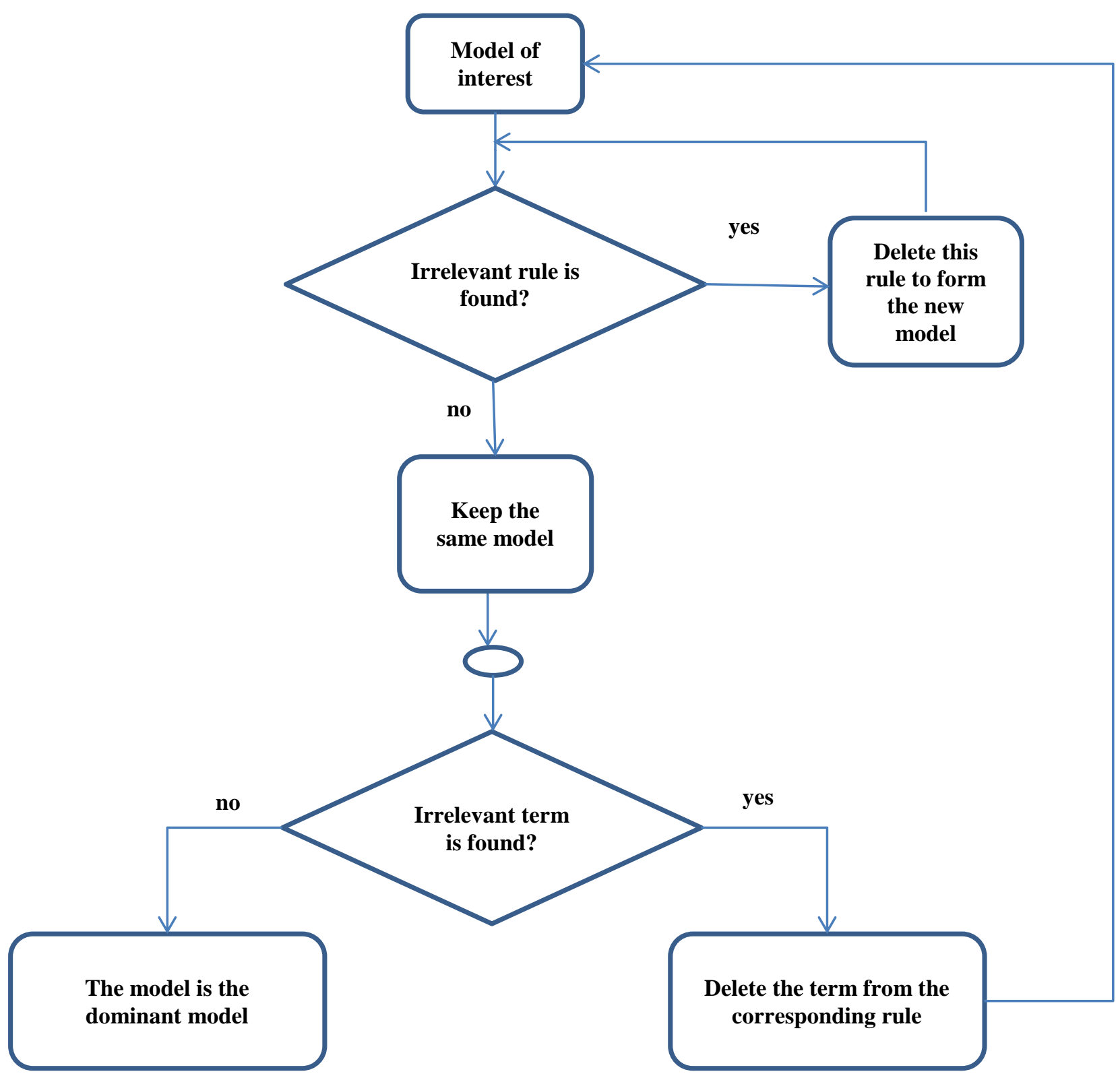


Figure 4: Decision chart for the search procedure for the dominant model

Consequently, model equivalency is verified by comparing the dominant models identified via the process just outlined. If the dominant models are the same, the models subjected to this comparison are equivalent.

\section{Theoretical Properties}

In this section, we present some theoretical properties. These properties distinguish equivalent models from non-equivalent models. We also prove the theorem that the dominant model is unique.

Property 1: Any rule in any equivalent model must be a child rule of a dominant rule or a dominant rule itself.

By definition, the dominant rules define the scope of the conditions for the defined class. Including any rule that is not a child rule of a dominant rule will introduce instance(s) beyond the scope of the equivalent models.

Property 2: The complement models of any equivalent models are also equivalent.

This property can be shown through graphical presentation (Figure 3). The three models are equivalent, with the area inside the three circles belonging, for example, to class 1 . The complement models for these three equivalent models cover the same area (outside the three circles) for class 0 . The three models and their complement models are clearly equivalent in terms of responses.

Theorem 1: The dominant model for each class is unique.

Proof: Suppose the dominant model is represented as

$$
\text { If } \bigcup_{i=1}^{d} \bigcap_{j=1}^{k_{i}} m_{i j} \text { then } \mathrm{Y}=1 \text {. }
$$

Note that $\mathrm{d}$ is the number of rules; $\mathrm{m} 1, \mathrm{~m} 2, \ldots, \mathrm{md}$ are the terms included in the conditions for rules $1,2, \ldots, \mathrm{d}$, respectively; and $\mathrm{k} 1, \mathrm{k} 2, \ldots, \mathrm{kd}$ are the numbers of the terms for the conditions for rules $1,2, \ldots, \mathrm{d}$, respectively. $\mathrm{Y}$ is the response, which is either 0 or 1 (we will use 1 , without loss of generality).

The complement model is then in the following form:

$$
\text { If } \bigcup_{\text {all } j_{1}, j_{2}, \ldots, j_{d}}\left(m_{1 j_{1}}{ }^{c} \cap m_{2 j_{2}}{ }^{c} \cap \ldots \cap m_{d j_{d}}{ }^{c}\right) \text {, then } \mathrm{Y}=0 \text {, }
$$

where $j_{1=1 \ldots \mathrm{k} 1}, j_{2}=1 \ldots \mathrm{k} 2$, and $j_{d}=1 \ldots \mathrm{kd}$. 
From property 1 , the rule in the equivalent models must be either the child rule of the dominant rule or the dominant rule itself. Therefore, the complement model of any equivalent model will be in the form

$$
\text { If } \bigcup_{\text {all } j_{1}, j_{2}, \ldots, j_{d}}\left(m_{1 j_{1}}{ }^{c} \cap m_{2 j_{2}}{ }^{c} \cap \ldots \cap m_{d j_{d}}{ }^{c}\right) \text { Uextra terms, then } \mathrm{Y}=0,
$$

where extra terms are the products of excessive condition terms.

From property 2, the complement models of the equivalent models will be equivalent.

This implies that Model (a) and Model (b) are equal. Thus, the extra terms, (Uextra terms ), are irrelevant and can be eliminated.

Note that we can further compress the term $\bigcup_{\text {all } j_{1}, j_{2}, \ldots, j_{d}}\left(m_{1 j_{1}}{ }^{c} \cap m_{2 j_{2}}{ }^{c} \cap \ldots \cap m_{d j_{d}}{ }^{c}\right)$ by eliminating any irrelevant terms and/or irrelevant rules to establish a dominant model for class 0 .

The term $\bigcup_{\text {all } j_{1}, j_{2}, \ldots, j_{d}}\left(m_{1 j_{1}}{ }^{c} \cap m_{2 j_{2}}{ }^{c} \cap \ldots \cap m_{d j_{d}}{ }^{c}\right)$ is unique; therefore, the dominant model is unique. This completes the proof of the theory.

Corollary. All the condition terms in the dominant model appear in all the equivalent models.

The importance of uniqueness suggests that all the equivalent models can refer to this dominant model. The proof implies that all the equivalent models can be compressed into their simplest final form, i.e., the dominant model. We propose to use this model as a benchmark for explaining the relationships among variables for all the equivalent models. An example of how to convert any model into its dominant model follows.

\section{Case Study: The MONK's dataset}

The MONK's dataset was compiled by Sebastian Thrun of Carnegie Mellon University using propositional formulas over six factors (Thrun et al 1991). It can be obtained from the UCI machine learning website (http://archive.ics.uci.edu/ml/datasets.html). The MONK's problem is the basis for the first international comparison of learning algorithms.

This dataset has been used in numerous studies to compare methodologies. Among these, some of the papers that focus on finding the classification error rate without considering the model are Philip and Joseph (2000), Karaçali and Krim (2003), Shin (2006), Naval and Yusiong (2007), Zhang and Wang (2008), Appavu and Rajaram (2009), Qodmanan et al. (2011). Additionally, other studies search for models that represent the dataset (Etchells and Lisboa 2006; Guerreiro and Trigueiros 2010; Marghny and El-Semman 2005). These papers focus on the underlined rules that are given to the dataset without considering other possible equivalent models. According to these studies, methodologies that discover the given rules are competent. The problem is that the methodology can be competent even when the underlined rules are not found, as other equivalent models can be found instead. In the literature, many methodologies 
have been studied in order to determine whether they can be used to find the given model (Etchells and Lisboa 2006; Guerreiro and Trigueiros 2010; Marghny and El-Semman 2005). We use the MONK's dataset to illustrate the effect of equivalent models with association rules. The derivation used to obtain the dominant model is also presented.

\subsection{Background of the MONK's Dataset}

The objective of the MONK's dataset is to classify 432 robots into two classes (class 0 and class 1) based on six attributes. The details of all the attributes are shown in Table 3. The given model assigns each robot to one of the two classes with a subset of the six attributes. Note that the given model for the MONK's dataset is designed on the basis of the following logic (the truth):

(1) If the head shape and the body shape of the robot are the same, then the robot is in class 1 , and

(2) If the robot wears a red jacket, then the robot is in class 1.

We convert the original attributes into dummy variables, as shown in Table 2 . The table also shows the role of equivalent rules in explaining the same dataset.

Table 2: Attributes for the MONK's Dataset

\begin{tabular}{|l|l|l|}
\hline Attribute & Level & Binary Variable \\
\hline head_shape & round, square, octagon & $\begin{array}{l}\mathrm{X}_{1}=1 \text { if head_shape is round, and } \mathrm{X}_{1}=0 \text { otherwise. } \\
\mathrm{X}_{2}=1 \text { if head_shape is square, and } \mathrm{X}_{2}=0 \text { otherwise. }\end{array}$ \\
\hline body_shape & round, square, octagon & $\begin{array}{l}\mathrm{X}_{3}=1 \text { if body_shape is round, and } \mathrm{X}_{3}=0 \text { otherwise. } \\
\mathrm{X}_{4}=1 \text { if body_shape is square, and } \mathrm{X}_{4}=0 \text { otherwise. }\end{array}$ \\
\hline is_smiling & yes, no & $\mathrm{X}_{5}=1$ if smiling, and $\mathrm{X}_{5}=0$ otherwise. \\
\hline holding & sword, balloon, flag & $\mathrm{X}_{6}=1$ if holding a sword, and $\mathrm{X}_{6}=0$ otherwise. \\
& & $\mathrm{X}_{7}=1$ if holding a balloon, and $\mathrm{X}_{7}=0$ otherwise. \\
\hline jacket_color & red, yellow, green, blue & $\mathrm{X}_{8}=1$ if jacket color is red, and $\mathrm{X}_{8}=0$ otherwise. \\
& & $\mathrm{X}_{9}=1$ if jacket color is yellow, and $\mathrm{X}_{9}=0$, otherwise. \\
& & $\mathrm{X}_{10}=1$ if jacket color is green, and $\mathrm{X}_{10}=0$ otherwise. \\
\hline has_tie & yes, no & $\mathrm{X}_{11}=1$ if wearing a tie, and $\mathrm{X}_{11}=0$ otherwise. \\
\hline
\end{tabular}

\subsection{Equivalent Models for the MONK's dataset}

If we follow the given logic with binary variables, the conditions are expressed in four rules - the dominant rules that form the dominant model, i.e., Model M, as shown in Table 3.

Table 3: Dominant Model of Class 1 for the MONK's Dataset (Model M)

\begin{tabular}{|l|l|}
\hline Rule & Explanation \\
\hline M1: If $X_{1}=1$ and $X_{3}=1$, then $Y=1$. & $\begin{array}{l}\text { If the robot has a round head shape and a round body } \\
\text { shape, then the robot is in class } 1 .\end{array}$ \\
\hline M2: If $X_{2}=1$ and $X_{4}=1$, then $Y=1$. & $\begin{array}{l}\text { If the robot has a square head shape and a square } \\
\text { body shape, then the robot is in class } 1 .\end{array}$ \\
\hline
\end{tabular}




\begin{tabular}{|l|l|}
\hline $\begin{array}{l}\text { M3: If } X_{1}=0, X_{2}=0, X_{3}=0, \text { and } X_{4}=0, \text { then } \\
Y=1 .\end{array}$ & $\begin{array}{l}\text { If the robot has an octagonal head shape and an } \\
\text { octagonal body shape, then the robot is in class } 1 .\end{array}$ \\
\hline M4: If $X_{8}=1$, then $Y=1$. & $\begin{array}{l}\text { If the robot wears a red jacket, then the robot is in } \\
\text { class } 1 .\end{array}$ \\
\hline
\end{tabular}

These four rules are the benchmark for comparing goodness of model and the criteria. Consider Model R, as shown in Table 4. There are seven rules, none of which appears in Model M (Table 3). Thus, by any existing criteria, Model R is not a good model. However, it can easily be verified that the responses generated by Model R are identical to those generated by Model M (Table 3). In other words, Model $\mathrm{R}$ is equivalent to Model $\mathrm{M}$.

Table 4: Equivalent Model for the MONK's Dataset (Model R)

\begin{tabular}{|c|c|}
\hline Rule & Explanation \\
\hline R1: If $X_{8}=1$ and $X_{1}=1$, then $Y=1$. & $\begin{array}{l}\text { If the robot wears a red jacket and has a round head } \\
\text { shape, then the robot is in class } 1 .\end{array}$ \\
\hline R2: If $X_{8}=0, X_{1}=1$, and $X_{3}=1$, then $Y=1$. & $\begin{array}{l}\text { If the robot has a round head shape, has a round } \\
\text { body shape, and does not wear a red jacket, then } \\
\text { the robot is in class } 1 \text {. }\end{array}$ \\
\hline R3: If $X_{8}=1$ and $X_{4}=1$, then $Y=1$ & $\begin{array}{l}\text { If the robot wears a red jacket and has a square } \\
\text { body shape, then the robot is in class } 1 .\end{array}$ \\
\hline R4: If $X_{8}=0, X_{2}=1$, and $X_{4}=1$, then $Y=1$ & $\begin{array}{l}\text { If the robot has a square head shape, has a square } \\
\text { body shape, and does not wear a red jacket, then } \\
\text { the robot is in class } 1 \text {. }\end{array}$ \\
\hline R5: If $X_{1}=0, X_{3}=0$, and $X_{8}=1$, then $Y=1$ & $\begin{array}{l}\text { If the robot does not have a round head shape, does } \\
\text { not have a round body shape, and wears a red } \\
\text { jacket, then the robot is in class } 1 \text {. }\end{array}$ \\
\hline $\begin{array}{l}\text { R6: If } X_{1}=0, X_{2}=0, X_{3}=0, X_{4}=0 \text {, and } X_{8}=0 \text {, } \\
\text { then } Y=1\end{array}$ & $\begin{array}{l}\text { If the robot has an octagonal head shape, has an } \\
\text { octagonal body shape, and does not wear a red } \\
\text { jacket, then the robot is in class } 1 \text {. }\end{array}$ \\
\hline R7: If $X_{1}=0, X_{4}=0$, and $X_{8}=1$, then $Y=1$ & $\begin{array}{l}\text { If the robot has a square head shape, has a square } \\
\text { body shape, and wears a red jacket, then the robot } \\
\text { is in class } 1 \text {. }\end{array}$ \\
\hline
\end{tabular}

These two models are equivalent in that they possess identical responses. However, they differ in regard to how they explain the relationships between input variables and responses.

\subsection{Dominant Model for MONK's Dataset}

For Model R, the original seven rules cannot be deleted in the first step. Therefore, we consider irrelevant terms. The term that can be deleted from Rule R1 is X1 $=1$, which leaves only the term X8 = 1 in Rule R1. Then Rule R3, Rule R5, and Rule R7 are deleted in the 
consecutive steps, as they become the child rules of the modified Rule R1. Next, the term X8 = 0 is deleted from Rule R2, which leaves only the terms $\mathrm{X} 1=1$ and X3 = 1 in Rule R2. Again, as there is no irrelevant rule, we consider the next irrelevant term. The term $\mathrm{X} 8=0$ is deleted from Rule R4, which leaves only terms X2 = 1 and X4 = 1 in Rule R4. As there is no other irrelevant rule we consider the next irrelevant term. The term X8 $=0$ is deleted from Rule R6, which leaves only the terms $\mathrm{X} 1=0, \mathrm{X} 2=0, \mathrm{X} 3=0$, and $\mathrm{X} 4=0$ in Rule R6. At this point, the dominant model, Model M, is obtained.

Note that the modified Rule R1 is identical to Rule M4, that the modified Rule R2 is identical to Rule M1, that the modified Rule R4 is identical to Rule M2, and that the modified Rule R6 is identical to Rule M3. As stated in Theorem 1, as this is the dominant model, it is unique. Note that we do not delete term X8 = 0 from Rule R2, Rule R4, and Rule R6 at the same time because deleting repeated terms does not always work with all equivalent models.

In the next section, we show that the issue of equivalent model can happen to any real-life applications illustrated through the Lenses dataset (Cendrowska 1987).

\section{Application: Lenses Dataset}

The Lenses dataset was donated by Benoit Julien and it can be obtained from the UCI machine learning website (http://archive.ics.uci.edu/ml/datasets.html). The objective of the Lenses dataset is to classify 24 patients into two classes (class 0: does not need contact lenses; and class 1: needs contact lenses) based on four attributes. Note that the original data classify the patients into three classes (those who need hard contact lenses, those who need soft contact lenses, and those who do not need contact lenses). We modified this dataset for the purpose of illustration. The details of all the attributes are shown in Table 5.

Table 5: Attributes for the Lense Dataset

\begin{tabular}{|l|l|l|}
\hline Attribute & Level & Binary Variable \\
\hline $\begin{array}{l}\text { Age of the } \\
\text { patient }\end{array}$ & $\begin{array}{l}\text { young, pre-presbyopic, } \\
\text { presbyopic }\end{array}$ & $\begin{array}{l}\mathrm{X}_{1}=1 \text { if young, and } \mathrm{X}_{1}=0 \text { otherwise. } \\
\mathrm{X}_{2}=1 \text { if pre-presbyopic, and } \mathrm{X}_{2}=0 \text { otherwise. }\end{array}$ \\
\hline $\begin{array}{l}\text { Spectacle } \\
\text { prescription }\end{array}$ & myope, hypermetrope & $\mathrm{X}_{3}=1$ if myope, and $\mathrm{X}_{3}=0$ otherwise. \\
\hline $\begin{array}{l}\text { Astigmatic } \\
\begin{array}{l}\text { Tear production } \\
\text { rate }\end{array}\end{array}$ & no, yes & $\mathrm{X}_{4}=1$ if no, and $\mathrm{X}_{4}=0$ otherwise. \\
\hline
\end{tabular}

To find a model for Lenses dataset, we use the CBA program (Liu et al. 1998) developed by the Department of Information Systems and Computer Sciences at the National University of Singapore (website: http://www.comp.nus.edu.sg/ dm2/). The model from the default setup by CBA is shown in Table 6.

Table 6: Model from association rules analysis for the Lenses Dataset 


\begin{tabular}{|l|l|}
\hline Rule & Explanation \\
\hline If $\mathrm{X}_{1}=1$ and $\mathrm{X}_{5}=0$, then $\mathrm{Y}=1$. & $\begin{array}{l}\text { If the patient is young and has a normal tear } \\
\text { production rate, then the patient should wear contact } \\
\text { lenses. }\end{array}$ \\
\hline $\begin{array}{l}\text { If } \mathrm{X}_{3}=1, \mathrm{X}_{4}=0, \text { and } \mathrm{X}_{5}=0, \\
\text { then } \mathrm{Y}=1 .\end{array}$ & $\begin{array}{l}\text { If the patient has a myope spectacle prescription, is } \\
\text { astigmatic, and has a normal tear production rate, } \\
\text { then the patient should wear contact lenses. }\end{array}$ \\
\hline $\begin{array}{l}\text { If } \mathrm{X}_{3}=0, \mathrm{X}_{4}=1, \text { and } \mathrm{X}_{5}=0, \\
\text { then } \mathrm{Y}=1 .\end{array}$ & $\begin{array}{l}\text { If the patient has a hypermetrope spectacle } \\
\text { prescription, is not astigmatic, and has a normal tear } \\
\text { production rate, then the patient should wear contact } \\
\text { lenses. }\end{array}$ \\
\hline If $\mathrm{X}_{3}=1$, and $\mathrm{X}_{5}=0$, then $\mathrm{Y}=1$. & $\begin{array}{l}\text { If the patient has a myope spectacle prescription and } \\
\text { has a normal tear production rate, then the patient } \\
\text { should wear contact lenses. }\end{array}$ \\
\hline
\end{tabular}

We show via the Lenses dataset that association rules analysis produces an irrelevant rule and irrelevant term. The second rule and the term X3 $=0$ in the third rule, as shown in Table 6 , will be eliminated to obtain the dominant model, as shown in Table 7.

Table 7: Dominant Model of Class 1 for the Lenses Dataset

\begin{tabular}{|l|l|}
\hline Rule & Explanation \\
\hline If $\mathrm{X}_{1}=1$ and $\mathrm{X}_{5}=0$, then $\mathrm{Y}=1$. & $\begin{array}{l}\text { If the patient is young and has a normal tear } \\
\text { production rate, then the patient should wear contact } \\
\text { lenses. }\end{array}$ \\
\hline If $\mathrm{X}_{4}=1$ and $\mathrm{X}_{5}=0$, then $\mathrm{Y}=1$. & $\begin{array}{l}\text { If the patient is not astigmatic and has a normal tear } \\
\text { production rate, then the patient should wear contact } \\
\text { lenses. }\end{array}$ \\
\hline If $\mathrm{X}_{3}=1$, and $\mathrm{X}_{5}=0$, then $\mathrm{Y}=1$. & $\begin{array}{l}\text { If the patient has a myope spectacle prescription and } \\
\text { has a normal tear production rate, then the patient } \\
\text { should wear contact lenses. }\end{array}$ \\
\hline
\end{tabular}

The illustration through the Lenses dataset shows the importance of obtaining the dominant model since the explanation from the dominant model in Table 7 is more sensible compared to the explanation from the model in Table 6.

Moreover, the phenomenon of equivalent models not only affects the association rules analysis, but it also affects the statistical techniques developed from the association rules analysis. For example, the model selection methodology for logistic regression by Changpetch and Lin (2013) performs variable selections among all the input variables and the variables generated from the selected association rules in order to build the optimal logistic regression model. Changpetch and Lin (2013) shows that the proposed methodology finds the dominant rules for the MONK's dataset. However, if the methodology finds the other equivalent models instead, the dominant model will be obtained by applying the procedure discussed in Section 3. 


\section{Parallel Effects to Decision Tree Analysis and the Statistical Model Selection}

\section{Method}

The effect of equivalent models is also relevant to decision tree analysis - a well-known method used to predict the class of response by decision rules. This technique has been used for many years with a wide range of applications (Yada et al. 2007; Wang and Liu 2008; Jaeger and Chen 2010; Lee 2010; Kim et al. 2011; Gepp et al. 2012; Delen et al. 2013).

Decision tree analysis often involves unavoidable irrelevant terms because of the hierarchical structure of this methodology. These irrelevant terms can appear in decision rules even with the most compact tree wherein the branches cannot be reduced any further. This study provides the methodology to establish the dominant model for decision tree analysis which is a different concept from that of finding the most compact decision tree (Quinlan 1986; Kienappel and Kneser 2001; Pal and Mather 2003; Pulkkinen and Koivisto 2008; Chandra and Varghese 2009; Cha and Tappert 2009). The present study shows that we can eliminate irrelevant terms or rules after obtaining the decision rules from the decision tree. Even though we obtain the most compact tree, the irrelevant rules and terms are tentative because of the hierarchical structure. To illustrate, we present examples of relevant terms and how to obtain the dominant models through the Lymphography dataset (Polat and Güneş 2007).

\subsection{Application: Lymphography Dataset}

In this section, we use the Lymphography dataset from the University Medical Centre at the Institute of Oncology in Ljubljana, Yugoslavia, which was graciously provided by M. Zwitter and M. Soklic. Here, we illustrate an application of our proposed method by eliminating the irrelevant terms from the decision rules.

The Lymphography dataset comprises records of lymph disease diagnoses in four classes: normal, metastases, malign lymph, and fibrosis. The dataset consists of 148 total instances: 2 normal diagnoses, 81 metastases diagnoses, 61 malign lymph diagnoses, and 4 fibrosis diagnoses. As there were so few normal and fibrosis diagnoses, we did not include them in our analysis. The modified dataset, therefore, comprised two classes and 142 instances. The goal was to determine the response classes for metastases and malign lymph. There were 18 numerically valued attributes, which we converted into binary variables, listed in Table 8 .

Table 8: Responses and Attributes for the Lymphography Dataset

\begin{tabular}{|l|l|l|}
\hline Attribute & Levels & Binary Variables \\
\hline Diagnosis class & malign lymph, metastases & $\begin{array}{l}\mathrm{Y}=1 \text { if the class is malign lymph } \\
\mathrm{Y}=0 \text { if the class is metastases }\end{array}$ \\
\hline Lymphatic & $\begin{array}{l}\text { arched, deformed, } \\
\text { displaced }\end{array}$ & $\begin{array}{l}\mathrm{X} 1=1 \text { if arched, and X1 }=0 \text { otherwise } \\
\mathrm{X} 2=1 \text { if deformed, and X2 }=0 \text { otherwise }\end{array}$ \\
\hline Block of afferent & yes, no & $\mathrm{X} 3=1$ if yes, and X3 =0 otherwise \\
\hline
\end{tabular}




\begin{tabular}{|c|c|c|}
\hline Block of lymph c & yes, no & $\mathrm{X} 4=1$ if yes, and $\mathrm{X} 4=0$ otherwise \\
\hline Block of lymph s & yes, no & $\mathrm{X} 5=1$ if yes, and X5 = 0 otherwise \\
\hline Bypass & yes, no & $\mathrm{X} 6=1$ if yes, and X6 = 0 otherwise \\
\hline Extravagates & yes, no & $\mathrm{X} 7=1$ if yes, and $\mathrm{X} 7=0$ otherwise \\
\hline Regeneration & yes, no & $\mathrm{X} 8=1$ if yes, and $\mathrm{X} 8=0$ otherwise \\
\hline Early uptake & yes, no & $X 9=1$ if yes, and $X 9=0$ otherwise \\
\hline $\begin{array}{l}\text { Lymph node } \\
\text { dimensions }\end{array}$ & 1,2 & $\mathrm{X} 10=1$ if 2 , and $\mathrm{X} 10=0$ otherwise \\
\hline $\begin{array}{l}\text { Lymph nodes } \\
\text { enlarged }\end{array}$ & $1,2,3,4$ & $\begin{array}{l}X 11=1 \text { if } 1, \text { and } X 11=0 \text { otherwise } \\
X 12=1 \text { if } 2, \text { and } X 12=0 \text { otherwise } \\
X 13=1 \text { if } 3 \text {, and } X 13=0 \text { otherwise }\end{array}$ \\
\hline Changes in lymph & bean, oval, round & $\begin{array}{l}\mathrm{X} 14=1 \text { if bean, and X14 }=0 \text { otherwise } \\
\mathrm{X} 15=1 \text { if oval, and X15 = } 0 \text { otherwise }\end{array}$ \\
\hline Defects in node & $\begin{array}{l}\text { no, lacunars, lacunars } \\
\text { marginal, lacunars central }\end{array}$ & $\begin{array}{l}\mathrm{X} 16=1 \text { if no, and X16 }=0 \text { otherwise } \\
\mathrm{X} 17=1 \text { if lacunars, and X17 }=0 \text { otherwise } \\
\mathrm{X} 18=1 \text { if lacunars marginal, and X18 =0 } \\
\text { otherwise }\end{array}$ \\
\hline Changes in node & $\begin{array}{l}\text { no, lacunars, lacunars } \\
\text { marginal, lacunars central }\end{array}$ & $\begin{array}{l}X 19=1 \text { if no, and X19=0 otherwise } \\
X 20=1 \text { if lacunars, and X20 = } 0 \text { otherwise } \\
\text { X21 }=1 \text { if lacunars marginal, and X21 = } \\
\text { otherwise }\end{array}$ \\
\hline $\begin{array}{l}\text { Changes in } \\
\text { structure }\end{array}$ & $\begin{array}{l}\text { no, grainy, drop-like, } \\
\text { coarse, diluted, reticular, } \\
\text { stripped, faint }\end{array}$ & - \\
\hline Special forms & no, chalices, vesicles & $\begin{array}{l}X 22=1 \text { if no, and X22=0 otherwise } \\
X 23=1 \text { if chalices, and X23=0 otherwise }\end{array}$ \\
\hline $\begin{array}{l}\text { Dislocation of } \\
\text { node }\end{array}$ & yes, no & $\mathrm{X} 24=1$ if yes, and X24 = 0 otherwise \\
\hline Exclusion of node & yes, no & $\mathrm{X} 25=1$ if yes, and $\mathrm{X} 25=0$ otherwise \\
\hline Number of nodes & $\begin{array}{l}0-9,10-19,20-29,30-39, \\
40-49,50-59,60-69 \\
>=70\end{array}$ & $\begin{array}{l}X 26=1 \text { if } 0-9, \text { and } X 26=0 \text { otherwise } \\
X 27=1 \text { if } 10-19, \text { and } X 27=0 \text { otherwise }\end{array}$ \\
\hline
\end{tabular}

In the original dataset, there are more levels within some variables. However, when we deleted the six instances of normal and fibrosis diagnoses, some levels were eliminated. We decided to eliminate the attribute change in structure, as we did not have a solid criterion on which to discretize it because it had eight levels within the binary variable. We discretized number of nodes by dividing the observations into three groups based on distribution characteristics.

Figure 5 shows one of the decision trees for the Lymphography dataset. With this decision tree, irrelevant terms are attached to the decision rules, as shown in Table 9. 


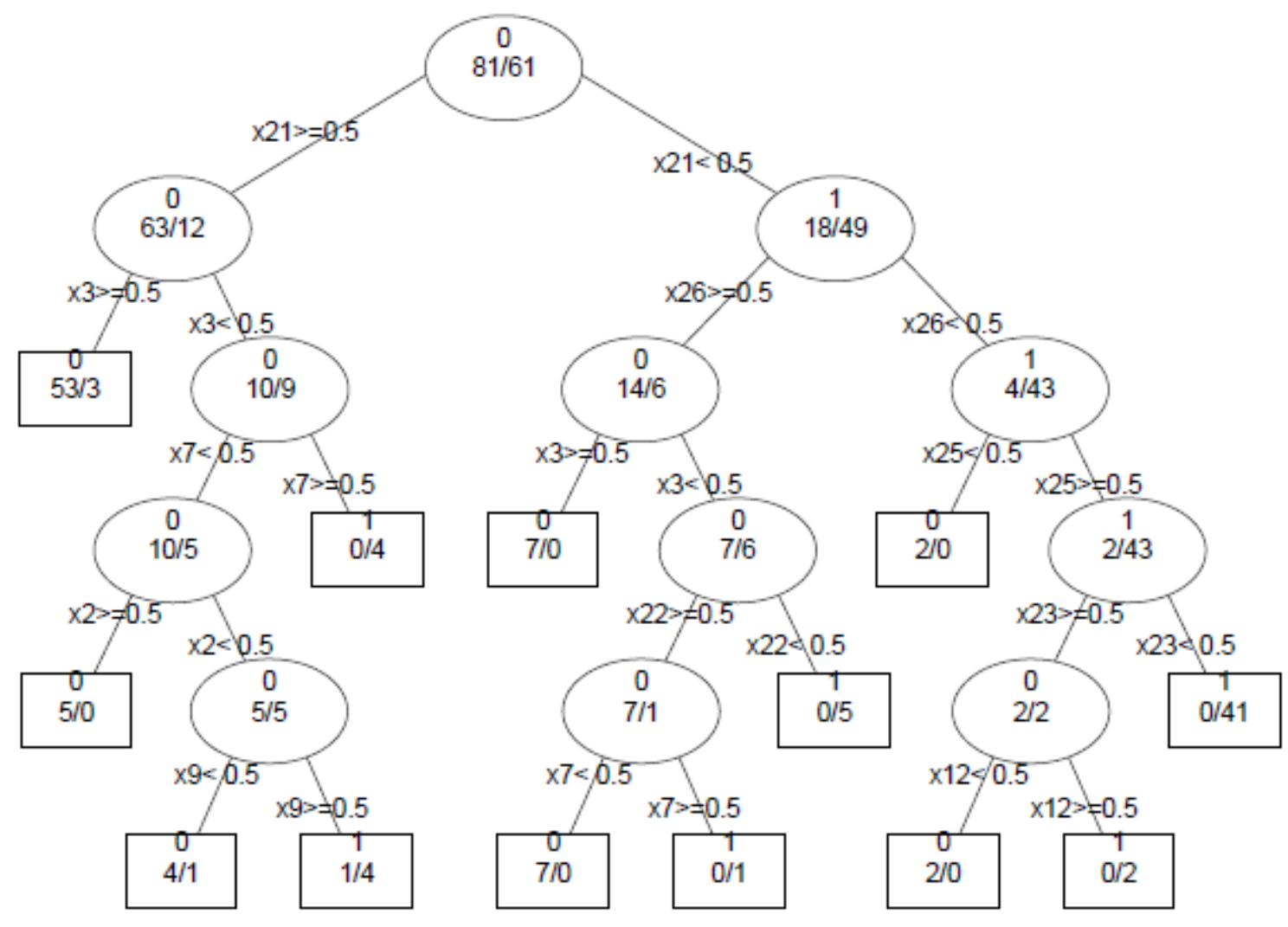

Figure 5: Decision tree for the Lymphography dataset

We show via the Lymphography dataset that decision tree analysis produces irrelevant terms. As shown in Table 9, the term X22 = 1 in the first rule, the term X23 = 1 in the third rule, and the term $\mathrm{X} 7=0$ in the sixth rule are eliminated to obtain the dominant model, as shown in Table 10 .

Table 9: Model from Decision Tree Analysis for the Lymphography Dataset

\begin{tabular}{|l|l|}
\hline Rule & Explanation \\
\hline $\begin{array}{l}\text { If } X_{21}=0, X_{26}=1, X_{3}=0, X_{22}=1, \text { and } \\
X_{7}=1, \text { then } Y=1 .\end{array}$ & $\begin{array}{l}\text { If changes in node is not lacunars marginal, } \\
\text { number of nodes is 0-9, block of afferent is no, } \\
\text { special forms is no, and extravagates is yes, then } \\
\text { the patient is in the malign lymph class. }\end{array}$ \\
\hline $\begin{array}{l}\text { If } X_{21}=0, X_{26}=1, X_{3}=0, \text { and } X_{22}=0, \\
\text { then } Y=1 .\end{array}$ & $\begin{array}{l}\text { If changes in node is not lacunars marginal, } \\
\text { number of nodes is 0-9, block of afferent is no, }\end{array}$ \\
\hline
\end{tabular}




\begin{tabular}{|l|l|}
\hline & $\begin{array}{l}\text { and special forms is either chalices or vesicles, } \\
\text { then the patient is in the malign lymph class. }\end{array}$ \\
\hline $\begin{array}{l}\text { If } \mathrm{X}_{21}=0, \mathrm{X}_{26}=0, \mathrm{X}_{25}=1, \mathrm{X}_{23}=1, \text { and } \\
\mathrm{X}_{12}=1, \text { then } \mathrm{Y}=1 .\end{array}$ & $\begin{array}{l}\text { If changes in node is not lacunars marginal, } \\
\text { number of nodes is not 0-9, exclusion of node } \\
\text { is yes, special forms is chalices, and lymph } \\
\text { nodes enlarged is 2, then the patient is in the } \\
\text { malign lymph class. }\end{array}$ \\
\hline $\begin{array}{l}\text { If } \mathrm{X}_{21}=0, \mathrm{X}_{26}=0, \mathrm{X}_{25}=1, \text { and } \mathrm{X}_{23}=0, \\
\text { then } \mathrm{Y}=1 .\end{array}$ & $\begin{array}{l}\text { If changes in node is not lacunars marginal, } \\
\text { number of nodes is not 0-9, exclusion of node } \\
\text { is yes, and special forms is not chalices, then the } \\
\text { patient is in the malign lymph class. }\end{array}$ \\
\hline $\begin{array}{l}\text { If } \mathrm{X}_{21}=1, \mathrm{X}_{3}=0, \text { and } \mathrm{X}_{7}=1, \text { then } \mathrm{Y}= \\
1 .\end{array}$ & $\begin{array}{l}\text { If changes in node is lacunars marginal, block } \\
\text { of afferent is no, and extravagates is yes, then } \\
\text { the patient is in the malign lymph class. }\end{array}$ \\
\hline $\begin{array}{l}\text { If } \mathrm{X}_{21}=1, \mathrm{X}_{3}=0, \mathrm{X}_{7}=0, \mathrm{X}_{2}=0, \text { and } \\
\mathrm{X}_{9}=1, \text { then } \mathrm{Y}=1 .\end{array}$ & $\begin{array}{l}\text { If changes in node is lacunars marginal, block } \\
\text { of afferent is no, extravagates is no, lymphatic } \\
\text { is not deformed, and early uptake is yes, then } \\
\text { the patient is in the malign lymph class. }\end{array}$ \\
\hline
\end{tabular}

Table 10: Dominant Model of Class 1 for the Lymphography Dataset

\begin{tabular}{|l|l|}
\hline Rule & Explanation \\
\hline $\begin{array}{l}\text { If } \mathrm{X}_{21}=0, \mathrm{X}_{26}=1, \mathrm{X}_{3}=0, \text { and } \mathrm{X}_{7}=1, \text { then } \\
\mathrm{Y}=1 .\end{array}$ & $\begin{array}{l}\text { If changes in node is not lacunars marginal, } \\
\text { number of nodes is 0-9, block of afferent is no, } \\
\text { and extravagates is yes, then the patient is in the } \\
\text { malign lymph class. }\end{array}$ \\
\hline $\begin{array}{l}\text { If } \mathrm{X}_{21}=0, \mathrm{X}_{26}=1, \mathrm{X}_{3}=0, \text { and } \mathrm{X}_{22}=0, \\
\text { then } \mathrm{Y}=1 .\end{array}$ & $\begin{array}{l}\text { If changes in node is not lacunars marginal, } \\
\text { number of nodes is 0-9, block of afferent is no, } \\
\text { special forms is either chalices or vesicles, then } \\
\text { the patient is in the malign lymph class. }\end{array}$ \\
\hline $\begin{array}{l}\text { If } \mathrm{X}_{21}=0, \mathrm{X}_{26}=0, \mathrm{X}_{25}=1, \text { and } \mathrm{X}_{12}=1, \\
\text { then } \mathrm{Y}=1 .\end{array}$ & $\begin{array}{l}\text { If changes in node is not lacunars marginal, } \\
\text { number of nodes is not 0-9, exclusion of node is } \\
\text { yes, and lymph nodes enlarged is 2, then the } \\
\text { patient is in the malign lymph class. }\end{array}$ \\
\hline
\end{tabular}




\begin{tabular}{|l|l|}
\hline $\begin{array}{l}\text { If } \mathrm{X}_{21}=0, \mathrm{X}_{26}=0, \mathrm{X}_{25}=1, \text { and } \mathrm{X}_{23}=0, \\
\text { then } \mathrm{Y}=1 .\end{array}$ & $\begin{array}{l}\text { If changes in node is not lacunars marginal, } \\
\text { number of nodes is not 0-9, exclusion of node is } \\
\text { yes, and special forms is not chalices, then the } \\
\text { patient is in the malign lymph class. }\end{array}$ \\
\hline If $\mathrm{X}_{21}=1, \mathrm{X}_{3}=0$, and $\mathrm{X}_{7}=1$, then $\mathrm{Y}=1$. & $\begin{array}{l}\text { If changes in node is lacunars marginal, block of } \\
\text { afferent is no, and extravagates is yes, then the } \\
\text { patient is in the malign lymph class. }\end{array}$ \\
\hline $\begin{array}{l}\text { If } \mathrm{X}_{21}=1, \mathrm{X}_{3}=0, \mathrm{X}_{2}=0, \text { and } \mathrm{X}_{9}=1, \text { then } \\
\mathrm{Y}=1 .\end{array}$ & $\begin{array}{l}\text { If changes in node is lacunars marginal, block of } \\
\text { afferent is no, lymphatic is not deformed, and } \\
\text { early uptake is yes, then the patient is in the malign } \\
\text { lymph class. }\end{array}$ \\
\hline
\end{tabular}

The application demonstrates that decision trees always generate irrelevant terms, which can be eliminated by our method. Accordingly, the dominant model can be found for any model from a decision tree.

As a consequence, the statistical techniques that use the variables generated from decision tree analysis will have this effect as well. For example, Kim (2009) combined the logistic regression model with decision tree analysis. Each branch of the decision tree becomes the variable (or cluster) for logistic regression model selection. Our approach can help to eliminate the irrelevant ones to formulate a consistent and concise explanation of the dataset.

\section{Conclusion}

The concept of model equivalency based on two or more models generating identical responses has not been adequately considered in the literature. Its effects are not only evident in association rules analysis, but also in decision tree analysis and other related statistical techniques. Our analysis shows that two models, which may not appear similar, can equally represent the same dataset. Therefore, failing to account for equivalency can lead to misleading results in regard to developing a methodology and making comparisons, as previously discussed.

On the other hand, the consequence of having different sets of rules to represent the same dataset is that we have more than one explanation for the dataset and these explanations may not be compatible. Our major finding is that although a large number of models may represent the same dataset, there is one unique model, i.e., the "dominant" model, to which all equivalent models can refer. The benefits of the dominant model are that it can be used as a benchmark to explain the relationships among the variables and to verify equivalency among models such that unfair comparisons among the rules selection methodologies and criteria are eliminated. 
This paper explored the phenomenon of equivalent models. First, we showed that misleading results are likely to be obtained if this phenomenon is not taken into consideration. Second, we considered and defined the concept of the dominant model among all equivalent models. We also elaborated our method for deriving the dominant model. Third, we proposed using the dominant model as the benchmark model for explaining the relationships between variables. We hope that this discovery will now be considered in association rules analysis, decision tree analysis, and other related data analysis and statistical methodologies.

Although we demonstrated the model equivalency phenomenon using predictive rules (supervised learning), these are generally class association rules (Li et al. 2001). We think it is likely that model equivalency is a factor in more general association models. 


\section{References}

[1] Agrawal, R. and Srikant, S. (1994). Fast algorithms for mining association rules. Proceedings of the ACM SIGMOD Conference on Management of Data, 487-499.

[2] Appavu, S. and Rajaram, R. (2009). Knowledge-based system for text classification using ID6NB algorithm. Knowledge-Based Systems 22: 1-7.

[3] Appice, A., Ceci, M., Lanza, A., Lisi, F. A., and Malerba, D. (2003). Discovery of spatial association rules in georeferenced census data: A relational mining approach. Intelligent Data Analysis 7(6): 541-566.

[4] Bastide, Y., Pasquier, N., Taouil, R., Stumme, G., and Lakhal, L. (2000). Mining minimal non-redundant association rules using frequent closed itemsets. Lecture Notes in Computer Science 1861: 972-986.

[5] Bayardo Jr, R. J. (1997). Brute-force mining of high-confidence classification rules. Proceedings of the Third International Conference on Knowledge Discovery and Data Mining, Newport Beach, CA, 123-126.

[6] Becquet, C., Blachon, S., Jeudy, B., Boulicaut, J. F., and Gandrillon, O. (2002). Strong association rule mining for large gene expression data analysis: A case study on human SAGE data. Genome Biology 3(12): 1-16.

[7] Cendrowska, J. (1987). PRISM: An algorithm for inducing modular rules. International Journal of Man-Machine Studies 27: 349-370.

[8] Cha, S. and Tappert, C. (2009). A generic algorithm for constructing compact binary decision trees. Journal of Pattern Recognition Research 1: 1-13.

[9] Chandra, B. and Varghese, P. P. (2009). Moving towards efficient decision tree construction. Information Sciences 179: 1059-1069.

[10] Changpetch, P. and Lin D. K. J. (2013). Model selection for logistic regression via association rules analysis. Journal of Computational Statistics and Simulations 83(8): 14151428.

[11] Delen, D., Kuzey, C. and Uyar, A. (2013). Measuring firm performance using financial ratios: A decision tree approach. Expert Systems with Applications 40: 3970-3983.

[12] Dong, G., Zhang, X., Wong, L. and Li, J. (1999). CAEP: Classification by aggregating emerging patterns. Proceedings of the Second International Conference on Discovery Science (DS'99), Japan, 30-42.

[13] Dopfer, K. and Potts, J. (2004). Evolutionary realism: A new ontology for economics. Journal of Economic Methodology 11: 195-212.

[14] Etchells, T. A. and Lisboa, P. J. G. (2006). Orthogonal Search-based Rule Extraction (OSRE) method for trained neural networks: A practical and efficient approach. IEEE Transactions on Neural Networks 17(2): 374-384.

[15] Garcia, P., Amandi, A., Schiaffino, S. and Campo, M. (2007). Evaluating Bayesian networks' precision for detecting students' learning styles. Computer and Education Journal 49: 794808.

[16] Gepp, A., Wilson, J. H., Kumar, K. and Bhattacharya, S. (2012). A Comparative Analysis of Decision Trees Vis-à-vis Other Computational Data Mining Techniques in Automotive Insurance Fraud Detection. Journal of Data Science 10(3): 537-561. 
[17] Grudzinski, K., Grochowski, M. and Duch, W. (2010). Pruning classification rules with reference vector selection methods. LNCS 6113: 347-354.

[18] Guerreiro, J. and Trigueiros, D. (2010). A unified approach to the extraction of rules from artificial neural networks and support vector machines. Advanced Data Mining and Applications, 34-42.

[19] Jaeger, S. and Chen, S. (2010). Information fusion for biological prediction. Journal of Data Science 8(2): 269-288.

[20] Karaçali, B. and Krim, H. (2003). Fast minimization of structural risk by nearest neighbor rule. IEEE Transactions on Neural Network 14(1), 127-137.

[21] Kienappel, A. K. and Kneser, R. (2001). Designing very compact decision trees for grapheme-to-phoneme transcription. In Proceedings Eurospeech (Scandinavia), 1911-1914.

[22] Kim, M. (2009). Two-stage logistic regression model. Expert Systems with Applications 36: $6727-6734$.

[23] Kim, S. S., Timothy, D. J. and Hwang, J. (2011). Understanding Japanese tourists' shopping preferences using the decision tree analysis method. Tourism management 32: 544-554.

[24] Kuo, R. J., Lin, S. Y. and Shih, C. W. (2007). Mining association rules through integration of clustering analysis and ant colony system for health insurance database in Taiwan. Expert Systems with Applications 33(3): 794-808.

[25] Lee, A. J. T., Hong, R. W., Ko, W. M., Tsao, W. K. and Lin, H. H. (2007). Mining spatial association rules in image databases. Information Sciences 177(7): 1593-1608.

[26] Lee, S. (2010). Using data envelopment analysis and decision trees for efficiency analysis and recommendation of B2C controls. Decision Support Systems 49: 486-497.

[27] Li, J. (2006). On optimal rule discovery. IEEE Transactions on Knowledge and Data Engineering 18(4): 460-471.

[28] Li, W., Han, J. and Pei, J. (2001). CMAR: Accurate and efficient classification based on multiple class-association rules. Proceedings of the 2001 International Conference on Data Mining (ICDM'01), San Jose, CA, 369-376.

[29] Liu, B., Hsu, W. and Ma, Y. (1998). Integrating classification and association rule mining. Proceedings of the Fourth International Conference on Knowledge Discovery and Data Mining (KDD'98), New York, 80-86.

[30] Liu, K. H., Weng, M. F., Tseng, C. Y., Chuang, Y. Y., Chen, M. S. (2008). Association and temporal rule mining for post-processing of semantic concept detection in video. IEEE TMM 10(2): 240-251.

[31] Marghny, M. H. and El-Semman, I. E. (2005). Extracting logical classification rules with gene expression programming: Microarray case study. Proceedings of the International Conference on Artificial Intelligence and Machine Learning (AIML'05), Cairo, Egypt, 1116.

[32] Matsumoto, K. (1998). An experimental agricultural data mining system. Proceedings of the First International Conference on Discovery Science (DS'98), Japan, 439-440.

[33] McIver, D. K. and Friedl, M. A, (2002). Using prior probabilities in decision tree classification of remotely sensed data. Remote Sensing of Environment 81: 253-261.

[34] Merceron, A. and Yacef, K. (2008). Interestingness measures for association rules in educational data. Proceedings of Educational Data Mining Conference (EDM'08), 57-66. 
[35] Mossong, J., Hens, N., Jit, M. et al. (2008). Social contacts and mixing patterns relevant to the spread of infectious diseases. PLoS Medicine 5(3): 381-391.

[36] Naval Jr, P. C. and Yusiong, J. P. T. (2007). An evolutionary multi-objective neural network optimizer with bias-based pruning heuristic. Advances in Neural Networks, 174-183.

[37] Pal, M. and Mather, P. M. (2003). An assessment of the effectiveness of decision tree methods for land cover classification. Remote Sensing of Environment 86: 554-565.

[38] Philip, N. S. and Joseph, K. B. (2000). Boosting the differences: A fast Bayesian classifier neural network. Intelligent Data Analysis 4: 463-473.

[39] Polat, K. and Güneş, S. (2007). Automatic determination of diseases related to lymph system from lymphography data using principles component analysis (PCA), fuzzy weighting preprocessing and ANFIS. Expert Systems with Applications 33: 636-641.

[40] Pulkkinen, P. and Koivisto, H. (2008). Fuzzy classifier identification using decision tree and multiobjective evolutionary algorithms. International Journal of Approximate Reasoning 48: 526-543.

[41] Qodmanan, H. R., Nasiri, M. and Minaei-Bidgoli, B. (2011). Multi objective association rule mining with genetic algorithm without specifying minimum support and minimum confidence. Expert Systems with Applications 38(1): 288-298.

[42] Quinlan, J. R. (1986). Induction of decision trees. Machine Learning 1, 81-106.

Shih, M. J., Liu, D. R. and Hsu, M. L. (2010). Discovering competitive intelligence by mining changes in patent trends. Expert Systems with Applications 37(4): 2882-2890.

[43] Shin, M. (2006). A performance study of Gaussian kernel classifiers for data mining applications. Advanced Data Mining and Applications: 189-196.

[44] Tesic, J., Newsam, S. and Manjunath, B. S. (2003). Mining image datasets using perceptual association rules. SIAM'03 Workshop on Mining Scientific and Engineering Datasets: 7177.

[45] Thabtah, F. (2007). A review of associative classification mining. The Knowledge Engineering Review 22(1): 37-65.

[46] Thrun, S. B., Bala, J., Bloedorn, E. et al. (1991). The MONK's problems-A performance comparison of different learning algorithms. Technical Report CS-CMU-91-197, Carnegie Mellon University, Pittsburgh, Pennsylvania

[47] Wang, C. and Liu, P. (2008). Data mining and hotspot detection in an urban development project. Journal of Data Science 6(3): 389-414.

[48] Wang, K., Zhou, S., Yang, Q. and Yeung, J. M. S. (2005). Mining customer value: From association rules to direct marketing. Data Mining and Knowledge Discovery 11(1): 57-79.

[49] Webb, G. I. and Zhang, S. (2005). K-optimal rule discovery. Data Mining and Knowledge Discovery 10(1): 39-79.

[50] Yada, K., Ip, E. and Katoh, N. (2007). Is this brand ephemeral? A multivariate tree-based decision analysis of new product sustainability. Decision Support Systems 44: 223-234.

[51] Yin, X. and Han, J. (2003). CPAR: Classification based on predictive association rules. Proceedings of the 2003 SIAM International Conference on Data Mining (SDM'03), San Francisco, CA, 331-335.

[52] Zhang, J. and Wang, Y. (2008). A rough margin based support vector machine. Information Sciences 178: 2204-2214. 
Pannapa Changpetch ${ }^{1}$

Department of Mathematical Sciences

Bentley University

175 Forest Street, Waltham, MA 02452, USA.

pchangpetch@bentley.edu

Dennis K. J. Lin ${ }^{2}$

Department of Statistics

Pennsylvania State University

University Park, PA 16802, USA.

DennisLin@psu.edu 